\title{
Short Circuit Calculation in Networks with a High Share of Inverter Based Distributed Generation
}

\author{
Harag Margossian, Juergen Sachau \\ Interdisciplinary Center for Security, Reliability and Trust \\ University of Luxembourg \\ Luxembourg City, Luxembourg
}

\author{
Geert Deconinck \\ ESAT-ELECTA \\ University of Leuven \\ Leuven, Belgium
}

\begin{abstract}
Conventional short circuit calculation programs do not consider the actual behavior of inverter based distributed generation (DG). Several techniques to consider them have been suggested in literature and are briefly described in this paper. A tool is developed with the combination of these techniques. The approach uses standard short circuit calculation tools and accommodates inverter based DG with different fault responses. The approach is evaluated and compared against other techniques using a realistic test distribution network model.
\end{abstract}

Keywords-inverter based distributed generation; short circuit analysis; grid code requirements

\section{INTRODUCTION}

Short circuit currents are analyzed during the planning and operation of a power system in order to facilitate the selection of electrical equipment and the design of the relaying and protection system. As the detailed modelling of the fault process can be too complicated when dealing with a large network, simple steady state solutions have been developed as a reasonable approximation [1]. Standard calculation methods simplify the network by applying symmetrical component theory such that grid elements like power lines, transformers, motors and generators are replaced by their effective impedances and make up a sequence network that can be used to analyze balanced and unbalanced faults [2].

The effective impedances for synchronous and asynchronous generators are based on the uncontrolled current output of these machines following a fault and can be classified according to the fault period of concern: subtransient, transient or steady state [3]. Inverter based distributed generation (DG) on the other hand have a controlled current output during faults; the inverter is decoupled from the grid and the short circuit current is determined by its control method instead of the type of the generator used. The inverter thus behaves more like a controlled current source than the ideal voltage source behind an impedance model used for synchronous and asynchronous generators [4]. It is therefore not straight-forward to accommodate inverter based DG in the standard short circuit calculation programs.

The requirement of DG to remain connected during faults and their significant levels of penetration in distribution networks mean their contribution to faults can no longer be

This work was conducted in collaboration with CREOS, the electricity network operator in Luxembourg and is supported by the National Research Fund, Luxembourg (C11/SR/1278568). neglected; hence several techniques have been suggested in literature to consider inverter based DG in fault calculation programs. These techniques range from using full time domain modelling that is very accurate but computationally heavy to more simple methods that sacrifice some accuracy for speed [5]. Focus in this paper is put on the latter type.

According to [6], it is possible to use the IEC 60909 provisions for reversible static converter-fed drives and treat the inverter based DG as asynchronous machines with a locked rotor to rated current ratio of 3 and an $\mathrm{R} / \mathrm{X}$ ratio of 0.1 . This results in an initial short circuit current of $3 \mathrm{pu}$ that decays to zero over time. The authors realize however that this is an overestimated initial value for the short circuit current produced by inverter based DG which also does not necessarily have a time decaying component. They consequently suggest considering an initial short circuit current of 1.5 to 2 times the rated current that remains constant over a predefined time period (100ms by default).

In [2] the authors suggest using the standard voltage source behind an impedance model for inverter based DG, where the effective impedance is calculated in such a way so as to control the resulting short circuit current to a pre-defined value. In their calculations the authors consider the worst case scenario where maximum current at a phase angle of $90^{\circ}$ is injected (i.e. purely reactive internal impedance). The negative and zero sequence impedances are considered as infinite (only positive sequence current is injected). The authors also propose using an iterative approach to achieve a more accurate result. It is however unlikely for all inverter based DG in a given grid to produce their maximum short circuit current following a fault. To account for the voltage dependency of the fault current produced and for the effect of pre-fault conditions, in [4], the authors suggest using look up tables to define a range of values for the short circuit current for every voltage level at the connection point of the DG.

In [7] the authors use the German Grid Code (GGC) requirements to determine the reactive and active current contributions of the inverter based DG and then use two iterative loops: an outer loop that calculates the resistance and phase angle of the voltage source to control the active current and an inner loop that calculates the reactance to control the reactive current. The approach uses the complete method for 
the short circuit calculation since the IEC and ANSI methods do not consider the phase angle of the voltage source.

This paper introduces a tool developed that uses a combination of the techniques mentioned and studies the impact of different approximations of the fault behavior of inverter based DG on fault currents and voltages in a given system. The approach is evaluated against dynamic simulations using Power System Simulator for Engineering (PSS/E) and the prospect of using the tool to evaluate grid code requirements is demonstrated.

\section{OVERVIEW OF THE CONSIDERED APPROACH}

The approach used to accommodate inverter based DG into short circuit calculation programs is illustrated in Figure 1. The algorithm consists of the following steps:

1. The nodal admittance matrix is constructed as in [1]. The inverter based DG are modeled as current sources and not using the voltage source behind an impedance model suggested in [2], thus impedances corresponding to these units need not be included in the admittance matrix. This is done to avoid recalculating and inverting the matrix in every iteration (when the inverter current values change).

2. The total fault current is calculated such that:

$$
I_{f t o t}=Z(f,:) * \vec{I} * \frac{-1}{Z(f, f)}
$$

where, $\mathrm{Z}$ is the impedance matrix, $\mathrm{f}$ is the fault node and I is the vector of node currents (including synchronous and inverter based DG). Pre-fault values for node voltages and inverter currents are considered (if not available, $\mathrm{Vi}=1$, $\mathrm{Ia}=$ Irated and $\mathrm{Ir}=0$ are used). The reason this is done as opposed to initially considering the inverters to be disconnected (similar to other approaches), is to get a better initial estimate. This is important, because the program allows modelling the DG to disconnect below certain voltages and an overall lower initial voltage level could lead to some DG being wrongfully considered disconnected.

3. The node voltages are calculated such that:

$$
\vec{V}=Z * \overrightarrow{I^{\prime}}
$$

where I' is the vector of node currents including the total fault current calculated in step 2 .

4. Based on the calculated voltages at the nodes where the inverter based DG are connected, short circuit active and reactive current setpoints are chosen. This is done from a database of current vs. voltage and (if available) vs. time curves (different inverters can have different models).

5. A check is performed to compare the active and reactive current setpoints to the values used in steps 2 and 3 . If the resulting error is within a chosen threshold, the algorithm is stopped; if not, it goes back to step 2 .

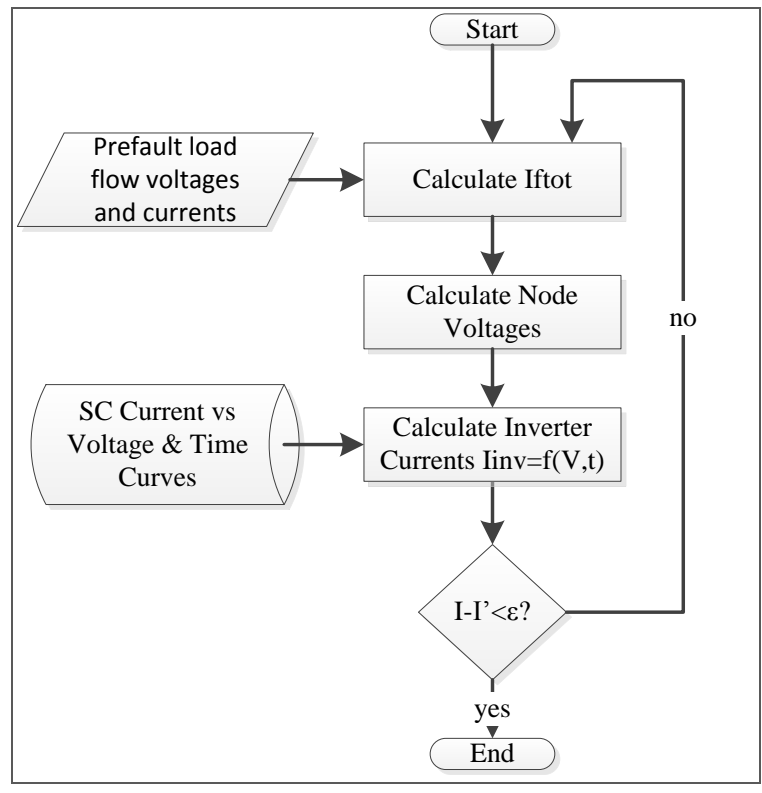

Fig. 1. Flowchart of Overall Approach

When current vs. time curves are available, the short circuit analysis tool can be run iteratively for every time period $\Delta t$. For every run of the algorithm after the first run, the initial short circuit calculation is skipped and the voltages calculated in the final iteration of the previous run are used instead (algorithm starts at step 4).

\section{SimULATION RESULTS}

A 61 node test distribution network is used in all the simulations presented in this paper. A simplified model showing the connection points of the inverter based DG and their relative positions to the substation can be seen in Figure 2.

The test network is characterized as follows:

- Voltage Level: $20 \mathrm{kV}$

- Two identical feeders with an average $\mathrm{X} / \mathrm{R}$ ratio of 1.38

- 8 MVA inverter based DG connected on each line

- Supply short circuit power of 500 MVA, with an R/X ratio of 0.26

- 31 MVA supply transformer with uk=12.5\%

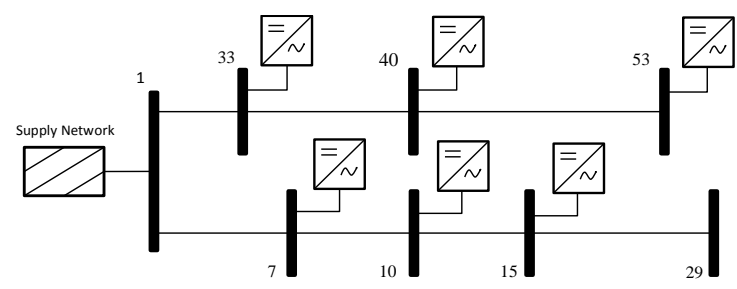

Fig. 2. Simplified One Line Diagram of Test Distribution Network 


\section{A. Validation with Dynamic Simulations}

In order to evaluate the developed tool, it is compared against dynamic simulations using PSS/E. The full power converter wind turbine model used in the simulations consists of the generator and electrical control models, WT4G2 and WT4E2 respectively. These models are available as part of the generic wind turbine model library.

The current vs. voltage curves of the wind turbines are approximated from a series of fault simulations and can be seen in Figure 3.

A snapshot at $100 \mathrm{~ms}$ following a three phase balanced fault at bus 29, simulated in PSS/E is then taken for comparison against the results of the short circuit analysis. The short circuit currents for some major lines in the test network can be seen in Table 1 and the voltages across the main nodes can be seen in Figure 4. While there are some discrepancies between the two results, they are generally quite small and acceptable.

Table 1: Short Circuit Currents Calculated in PSS/E vs MATLAB

\begin{tabular}{|c|c|c|c|}
\hline Line & $\mathbf{I}_{\text {PSS/E }}(\mathbf{p u})$ & $\mathbf{I}_{\text {MATLAB }}(\mathbf{p u})$ & \%Difference \\
\hline $1-7$ & 5.011 & 5.074 & 1.257 \\
\hline $7-10$ & 5.519 & 5.578 & 1.069 \\
\hline $10-15$ & 5.775 & 5.827 & 0.9 \\
\hline $15-29$ & 6.031 & 6.076 & 0.746 \\
\hline $33-1$ & 0.852 & 0.998 & 17.1 \\
\hline
\end{tabular}

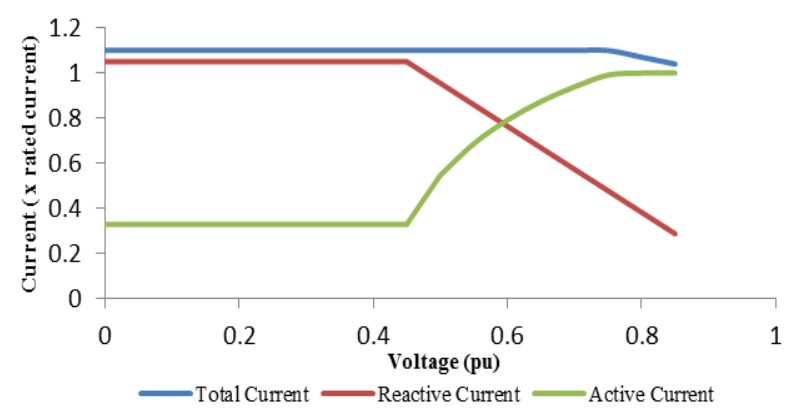

Fig. 4. Fault Current vs Voltage Curves for PSS/E Model

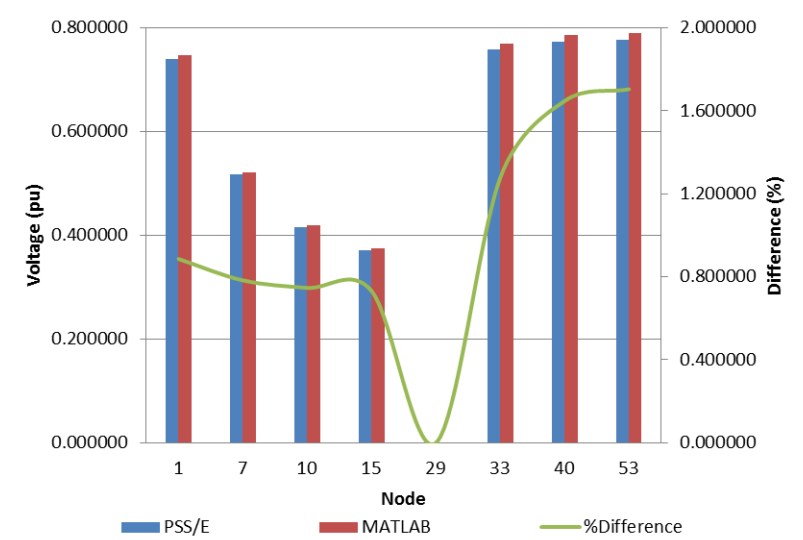

Fig. 5. Node Voltages Calculated in PSS/E vs MATLAB

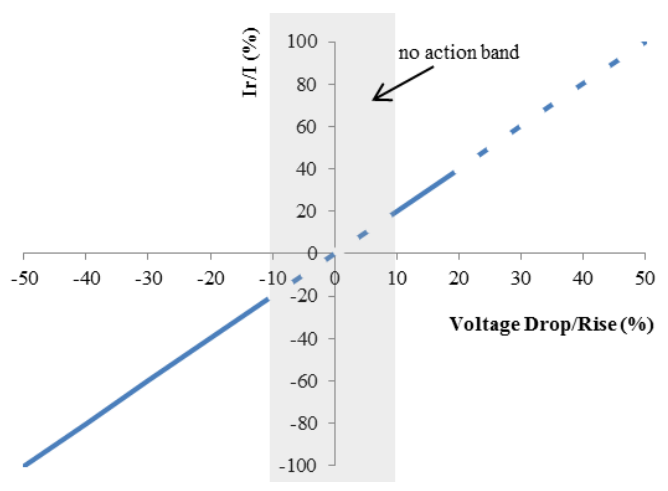

Fig. 5. Reactive Current Injection requirements for trhe German Grid Code

\section{B. Inverter Response Approximations}

To assess the impact different approximations of the fault current produced by inverter based DG have, the same test network is analyzed using three inverter control models (current vs. voltage curves) for the same three phase balanced fault on bus 29. The maximum current the inverters can produce is set to 1.5 times their rated current.

In the first case, the contribution of the DG to the fault is assumed to be in line with the German Grid Code requirements for distribution networks [8]. The required reactive current depends on the voltage and is given by the curve seen in Figure 5. For voltages below $0.5 \mathrm{pu}$, the reactive current is equal to the nominal current.

In the second case a maximum reactive current injection is considered (same as in [2]) and in the third, the contribution is completely neglected. The short circuit currents for some major lines in the test network can be seen in Figure 6 and the voltages across the main nodes can be seen in Figure 7 .

The total fault current coming from all sources is the current in line 15-29. Neglecting the fault current from the DG (case 3) leads to a 15\% lower value, while overestimating their contribution by assuming maximum current injection (case 2) results in a $6 \%$ higher value than when the GGC model is considered (case 1). These results were expected, because higher contributions from the DG result in a higher overall fault current.

Looking at the fault current at the level of the substation (line 1-7), the results are less obvious. For both cases 1 and 2, the fault current seen at the substation is higher than in case 3 by around $5 \%$. There are two main factors that cause this difference:

- The current coming from the DG connected to the healthy feeder.

- The fault contributions of the DG connected to the feeder with the fault, which mask the total fault current (an effect known as the blinding of protection [9]).

When comparing case 2 to case 1 , the effect of (a) is higher than (b) and when comparing case 3 to case 1 , the effect of (b) is higher than (a), resulting in a higher current in both comparisons. 


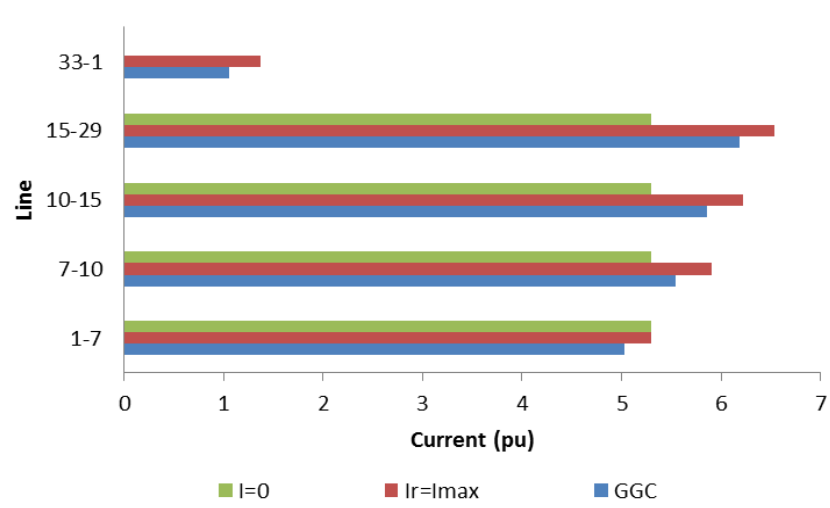

Fig. 6. Calculated Short Circuit Currents for Different Inverter Control Models

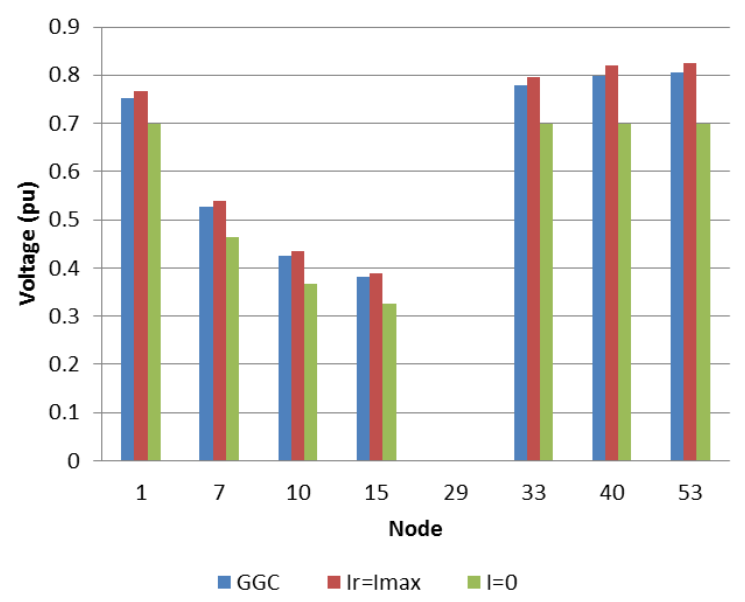

Fig. 7. Calculated Node Voltages for Different Inverter Control Models

Looking at the node voltages, the results for the three cases are as expected. For case 2, the voltages are higher than in case 1 with the biggest difference at node 1 being $0.035 \mathrm{pu}(4.5 \%)$. For case 3 , the voltages are lower than in case 1, with the biggest difference at node 7 being $0.065 \mathrm{pu}(12 \%)$.

Different network configurations can be more favorable for different approximations of the fault contribution of inverter based DG. Since multiple factors affect the results of the short circuit analysis, it is best to use the most accurate models possible without over-complicating the calculations.

\section{Evaluating Grid Code Requirements}

The fault ride through (FRT) requirements of the German Grid Code for inverter based DG connected to the distribution network are illustrated in Figure 8 and can be summarized as follows [8]:

- Above limit 1, the plant must remain connected and provide dynamic voltage support as in stable operation (Figure 8).

- Between limits 1 and 2, it is to be discussed with the Distribution Network Operator (DNO)

- Below limit 2, instant disconnection is accepted

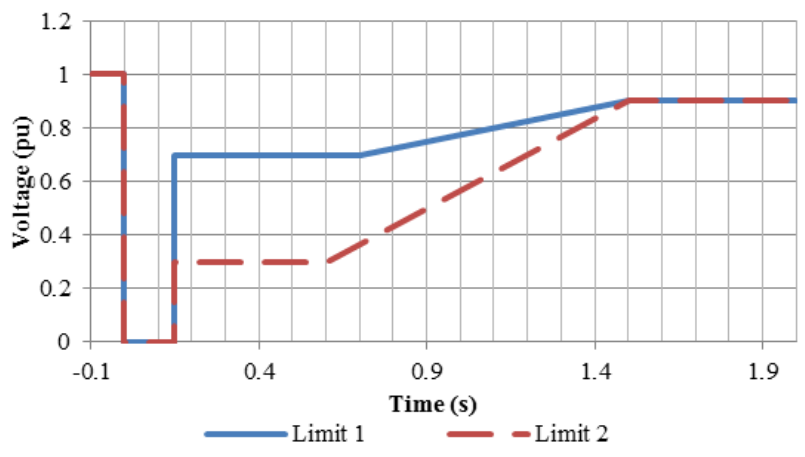

Fig. 8. GGC FRT Requirements for Inverter Based DG

In order to compare the effect of choosing limit 2 over limit 1 for the test distribution network, the short circuit analysis tool is run for every $50 \mathrm{~ms}$ after a three phase balanced fault on bus 29. The calculated total short circuit current and the current seen at the substation, when considering limit 1 or limit 2 as the inverter control model, are illustrated in Figure 9.

Choosing limit 2 over limit 1 leads to a higher total short circuit current, due to the inverter based DG remaining connected for lower voltages. But looking at the fault current at the level of the substation (where the overcurrent relay protecting the feeder is located), it is higher for limit 1 than limit 2 from $150 \mathrm{~ms}$ to $900 \mathrm{~ms}$ after the fault. This is because the
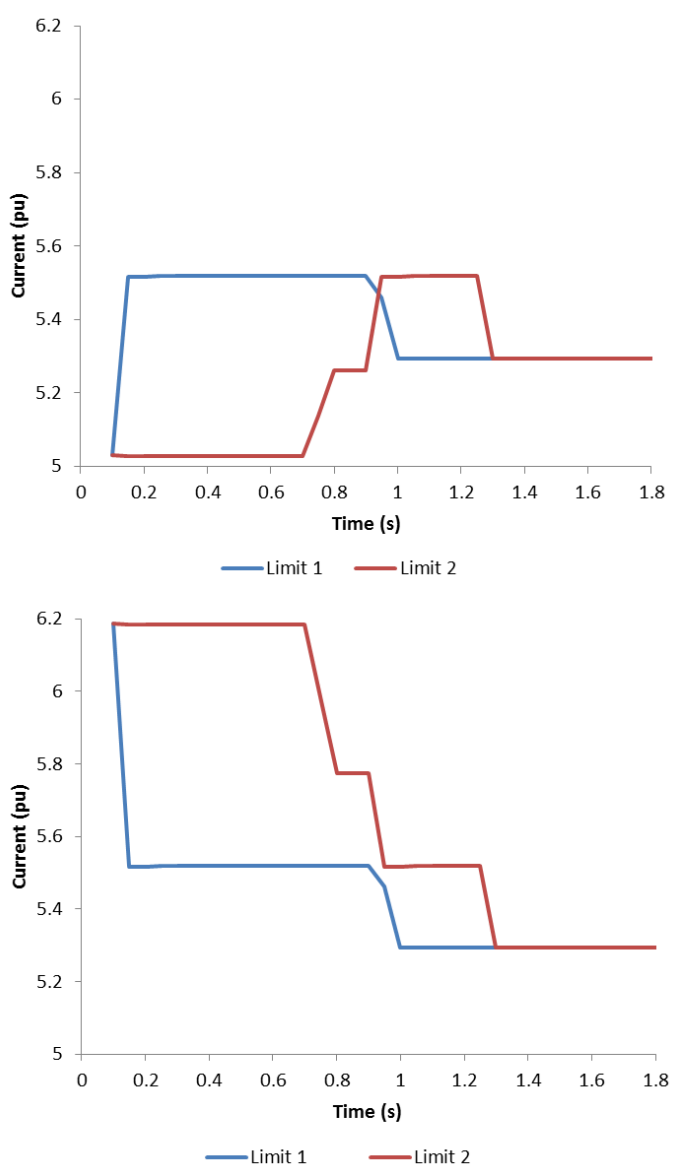

Fig. 9. Calculated Short Circuit Currents for GGC (left: total fault current; right: fault current at substation) 
DG connected to the feeder with the fault, disconnect after $150 \mathrm{~ms}$ when considering limit 1 but not limit 2 . Thus, the blinding effect is less for the case with limit 1 in that time period.

When choosing grid code requirements there are many more factors to consider, including voltage levels and different types of faults occurring at different locations. The short circuit analysis program presented in this paper can be used as a quick assessment tool. It can also be used to help set grid requirements progressively such that new DG that are to be connected to a particular network are required to ride through lower voltages or disconnect sooner than already connected DG.

\section{CONCLUSION}

Inverter based DG react differently to faults than synchronous or asynchronous generators. The fault currents they produce depend on the control method used and cannot be simulated with a simple voltage source behind an impedance model. This complicates the application of short circuit calculation standards.

In this paper, a short circuit analysis approach that accommodates different fault responses of inverter based DG has been presented. The approach is based on several techniques from literature and builds on standard tools. It has been evaluated against dynamic simulations in PSS/E with satisfactory results. The impact of different approximations of the fault response on the calculated short circuit currents and voltages has also been demonstrated. There are many factors that could cause an approximation to result in an over or underestimation of different parameters. Choosing a "safe" approximation thus depends on the network configuration and the parameter to be studied. Finally, the effect of choosing different FRT requirements for inverter based DG on short circuit currents has been illustrated. The possibility to use the short circuit analysis tool to impose progressively different requirements on new DG has been highlighted.

\section{REFERENCES}

[1] A. Gomez-Exposito, A. J. Conejo and C. Canizares, 2008, Electric Energy Systems: Analysis and Operation, CRC Press.

[2] M. Fischer and A. Mendonca, "Representation of Variable Speed Full Conversion Wind Energy Converters for Steady State Short-Circuit Calculations", in IEEE Power and Energy Society General Meeting, 2011, pp. 1-4.

[3] R. J. Nelson, "Short Circuit Contributions of Full Converter Wind Turbines", in IEEE PES Transmission and Distribution Conference and Exposition, 2012.

[4] R. A. Walling, E. Gursoy and B. English, "Current Contributions from Type 3 and Type 4 Wind Turbine Generators During Faults", in IEEE PES Transmission and Distribution Conference and Exposition, 2012.

[5] T. Wijnhoven, J. Tant and G. Deconinck, "Inverter Modelling Techniques for Protection Studies", in 3rd IEEE international Symposium on Power Electronics for Distributed Generation Systems (PEDG), 2012, pp. 187-194.
[6] T. N. Boutsika and S. A. Papathanassiou, "Short-Circuit Calculations in Networks with Distributed Generation", in Electric Power Systems Research, 78, 2008, pp. 1181-1191.

[7] M. Valentini, V. Akhmatov, F. Iov and J. Thisted, "Fault Current Contributions from VSC-based WindTurbines to the Grid", in 2nd International Symposium on Electrical and Electronics Engineering (ISEEE), 2008.

[8] A. Notholt, "Germany's New Code for Generation Plants Connected to Medium-Voltage Networks and its Repercussion on Inverter Control", in International Conference on Renewable Energies and Power Quality (ICREPQ), 2009.

[9] H. Margossian, F. Capitanescu and J. Sachau, "Feeder Protection Challenges with High Penetration of Inverter Based Distributed Generation", in IEEE International Conference on Computer as a tool (EUROCON), 2013, pp. 1369-1374. 